\title{
A adequação do sistema de Tableaux TLK
}

\author{
Helen G. da Silva*, Luiz Henrique da C. Silvestrini \\ Departamento de Matemática, FC, UNESP \\ 17033-360, Bauru, SP \\ E-mails: silvestrini@fc.unesp.br; helen-277@hotmail.com
}

\begin{abstract}
RESUMO
No presente trabalho, utilizamos o sistema de tableaux analíticos TLK, introduzido em [5], o qual foi construído como um sistema dedutivo alternativo à Lógica do Poucos, apresentado por Oliveira em 2011. Esta lógica, ao propor uma formalização para o quantificador 'poucos' da linguagem natural, é construída inspirada em um sistema axiomático modulado, segundo o qual a noção de 'muitos' é internalizada em nível da linguagem objeto. Desse modo, estabeleceremos a adequação do sistema TLK, ou seja, demonstraremos a correção e completude desse novo sistema em relação à Lógica do Poucos.
\end{abstract}

Palavras-chave: Lógicas Moduladas, Tableaux Analíticos.

\section{Introdução}

A Teoria da Prova constitui-se como um domínio de investigação avançado da Lógica, e ainda, compreendida como demonstração automática de teoremas consolida-se como uma profícua subárea da Teoria da Computação. $\mathrm{O}$ estudo das propriedades estruturais de provas formais constitui o cerne da pesquisa relacionada à Teoria da Prova, que por sua vez está relacionada com o conceito de decidibilidade desde os tempos de David Hilbert (1862-1943).

Os sistemas de provas, introduzidos por Gerhard Gentzen em [2], eram caracterizados por admitir o princípio das subfórmulas. Além disso, a teoria da prova desenvolvida por Gentzen consistia em demonstrar a validade de um argumento de uma maneira usualmente mais rápida, apenas trabalhando com regras em métodos finitários. Esses sistemas de provas são hoje conhecidos como Dedução Natural e Cálculo de Sequentes.

Os métodos de dedução criados por Gentzen culminaram no surgimento do método de tableaux, o qual também estabelece estruturas que permitem a representação e a dedução formal de conhecimento. Um tableau é mais adequado, em relação ao sistema axiomático, para implementações em computadores, pois este pode ser definido como uma árvore ordenada diádica.

Smullyan, em 1968 (cf. [6]), cunhou o termo tableaux analíticos. Este método dedutivo pode ser considerado uma variante dos métodos de Hintikka, como destaca o próprio Smullyan (1968, p. 15).

Um sistema de tableaux é apresentado apenas com regras de expansão ou regras para a construção dos tableaux como uma árvore ordenada diádica, as quais permitem a análise das fórmulas de uma linguagem $\mathcal{L}$ (cf. [1]). A noção de expansão é justamente expandir um ramo de um tableau. Empregamos a palavra "ramo" para designar um caminho ou uma possibilidade de análise das fórmulas dadas.

Por outro lado, a Lógica do Poucos, introduzida por Oliveira em [4], foi inspirada em uma dualização para uma lógica modulada que formaliza o quantificador 'muitos' da linguagem natural. A noção de quantificador será utilizada aqui como em [3].

Silva e Silvestrini em [5] propuseram o sistema de tableaux TLK, caracterizado como um sistema dedutivo alternativo ao axiomático apresentado em [4]. Neste trabalho, propomos explicitar a correção e completude do sistema TLK proposto em [5].

\footnotetext{
* Bolsista de Iniciação Científica. Processo FAPESP nº 2012/10272-5.
} 


\section{Materiais e Métodos}

Trata-se de um trabalho teórico e a presente pesquisa possibilita reconhecer o método de tableaux como um método alternativo ao axiomático, dessa maneira, utilizamos o método das árvores ordenadas diádicas para definir uma sequência de tableau e explicitamos os teoremas necessários para estabelecer a adequação do sistema TLK.

\section{Objetivo}

Demonstrar a correção e completude do sistema de tableaux TLK, em relação à Lógica do Poucos, introduzida por Oliveira em [4].

\section{Resultados e Discussão}

Consideremos que no sistema de tableaux TLK, $\Gamma \Vdash \varphi$ denota que a fórmula $\varphi$ é consequência analítica (i.e., existe um tableau fechado para $\varphi$ ) a partir de um conjunto $\Gamma$ de fórmulas, e ainda, que no sistema hilbertiano da Lógica do Poucos (LP), denotamos que $\varphi$ é uma consequência sintática de $\Gamma$, por $\Gamma \vdash \varphi$, de acordo com as definições da lógica clássica, ou seja, existe uma demonstração de $\varphi$ a partir de $\Gamma$, via axiomas da Lógica do Poucos. A partir disso, podemos demonstrar que $\Gamma \vdash \varphi$ se, e somente se, $\Gamma \Vdash \varphi$. Desse modo, todas as sentenças demonstradas na LP, também são demonstradas no sistema TLK, e vice-versa. Por fim, uma vez que a LP foi demonstrada ser correta e completa em [4], então nosso sistema também será correto e completo.

\section{Considerações Finais}

Abordamos neste trabalho a Lógica do Poucos em um sistema de tableaux analíticos introduzido via regras de expansão conforme apresentado em [5]. Em seguida, demonstramos alguns teoremas para estabelecer a equivalência dedutiva entre o sistema axiomático de Oliveira e o nosso sistema TLK. Dessa maneira, demonstramos que $\Gamma \Vdash \varphi \Leftrightarrow \Gamma \vdash \varphi$, e nesse sentido, estabelecemos a equivalência entre as consequências lógicas de cada sistema dedutivo apresentado. Uma vez que Oliveira em [4] demonstrou a correção e completude do sistema axiomático de LP, se $\Gamma \vDash \varphi$ denota que $\varphi$ é consequência semântica de $\Gamma$, sabemos que $\Gamma \vdash \varphi \Leftrightarrow$ $\Gamma \vDash \varphi$, consequentemente, o sistema de tableaux TLK também será correto e completo.

\section{Referências}

[1] M. C. Fitting, Introduction. In: M. D’Agostino; D. V. Gabbay; R. Hahnle; J. Posegga (Eds.). Handbook of Tableaux Methods. Dordrecht: Kluwer Academic Publishers, p. 1- 43, 1999.

[2] G. Gentzen, Untersuchungen über das logische Schlieben. Mathematische Zeitschrift. v. 39. 1935.

[3] A. Mostowski, On a generalization of quantifiers. Fundamenta Mathematicae, 44:12-36, 1957.

[4] K. E. C. S. Oliveira, Uma lógica do poucos. Dissertação de Mestrado. FFC-UNESP. Marília, 2011.

[5] H. G. da Silva; L. H. C. Silvestrini. Tableaux para uma lógica quase-modulada do 'poucos'. Anais do II Congresso de Matemática Aplicada e Computacional - Sudeste. Bauru, 2013.

[6] R. M. Smullyan. First-order logic. New York: Springer-Verlag/Dover Publication, 1968. 